\title{
Pathway and network-based analysis of genome-wide association studies and RT-PCR validation in polycystic ovary syndrome
}

\author{
HAORAN SHEN ${ }^{1}$, ZHOU LIANG ${ }^{2}$, SAIHUA ZHENG $^{1}$ and XUELIAN LI ${ }^{1,3}$ \\ ${ }^{1}$ Department of Gynecology, Obstetrics and Gynecology Hospital of Fudan University, Shanghai; \\ ${ }^{2}$ Department of Assisted Reproduction, Shanghai Ninth People's Hospital, Shanghai Jiaotong University School of Medicine, \\ Shanghai; ${ }^{3}$ Shanghai Key Laboratory of Female Reproductive Endocrine-Related Diseases, Shanghai, P.R. China
}

Received July 4, 2016; Accepted September 7, 2017

DOI: $10.3892 /$ ijmm.2017.3146

\begin{abstract}
The purpose of this study was to identify promising candidate genes and pathways in polycystic ovary syndrome (PCOS). Microarray dataset GSE345269 obtained from the Gene Expression Omnibus database includes 7 granulosa cell samples from $P C O S$ patients, and 3 normal granulosa cell samples. Differentially expressed genes (DEGs) were screened between PCOS and normal samples. Pathway enrichment analysis was conducted for DEGs using ClueGO and CluePedia plugin of Cytoscape. A Reactome functional interaction (FI) network of the DEGs was built using ReactomeFIViz, and then network modules were extracted, followed by pathway enrichment analysis for the modules. Expression of DEGs in granulosa cell samples was measured using quantitative RT-PCR. A total of 674 DEGs were retained, which were significantly enriched with inflammation and immune-related pathways. Eight modules were extracted from the Reactome FI network. Pathway enrichment analysis revealed significant pathways of each module: module 0, Regulation of RhoA activity and Signaling by Rho GTPases pathways shared ARHGAP4 and ARHGAP9; module 2, GlycoProtein VI-mediated activation cascade pathway was enriched with RHOG; module 3, Thromboxane A2 receptor signaling, Chemokine signaling pathway, CXCR4-mediated signaling events pathways were enriched with LYN, the hub gene of module 3. Results of RT-PCR confirmed the finding of the bioinformatic analysis that ARHGAP4, ARHGAP9, RHOG and LYN were significantly upregulated in PCOS. RhoArelated pathways, GlycoProtein VI-mediated activation cascade pathway, ARHGAP4, ARHGAP9, RHOG and LYN may be involved in the pathogenesis of PCOS.
\end{abstract}

Correspondence to: Dr Xuelian Li or Dr Haoran Shen, Department of Gynecology, Obstetrics and Gynecology Hospital of Fudan University, 419 Fangxie Road, Shanghai 200011, P.R. China

E-mail: xllifc@fudan.edu.cn

E-mail: alainshen@aliyun.com

Key words: polycystic ovary syndrome, differentially expressed genes, pathway, network

\section{Introduction}

Polycystic ovary syndrome (PCOS) is a complex endocrine disorder caused by hormone imbalance and often presents reproductive, metabolic and psychological syndromes (1). The reproductive syndromes mainly include ovulatory dysfunction, hyperandrogenism and polycystic ovaries, and the metabolic syndromes primarily include insulin resistance, obesity and dyslipidemia $(2,3)$. The prevalence of PCOS is $\sim 18 \%$ in women of productive age based on Rotterdam diagnostic criteria (4). To date, there is no cure for PCOS, and treatment and interventions tailored to clinical features are recommended. The etiology of PCOS is complex and remains largely unknown. Genetic and environmental factors are two primary contributors to the disorder (5). Exploration of the molecular mechanisms of PCOS will promote the understanding of its pathogenesis and has important implications for designing novel therapy.

Molecular mechanisms of PCOS have been increasingly investigated. It was recently found that hypoxia-inducible factor (HIF)-1 $\alpha$-mediated endothelin (ET)-2 signaling was suppressed in a PCOS mouse model and closely associated with the development of PCOS (6). Moreover, microarray and bioinformatic analysis have been utilized to unravel the molecular mechanisms of PCOS and yielded considerable results. For example, dysregulated circulating miRNAs have been predicted to be associated with several signaling pathways, such as immune, angiogenesis and p53 signaling in PCOS (7). In addition, there is evidence that Notch signaling and mitogen activated protein kinase (MAPK) pathways may be involved in the progression of PCOS (8). Furthermore, it has been suggested that the dysregulated genes between PCOS patients with and without insulin resistance may play roles in PCOS-related metabolic abnormalities and follicular growth arrest (9). Liu et al applied a sub-pathway method to identify candidate agents for PCOS treatment (10), and studied the transcription factor-microRNA synergistic regulatory network in PCOS (11) based on the transcript profile GSE34526. Additionally, this dataset was used by Bohler et al to collaborate the WikiPathways and Reactome as a new analysis tool of different omics datasets (12). Despite of these achievements, the molecular mechanisms of PCOS remain unclear. 
It has been demonstrated that network-based data could offer an integrated view of the genes or proteins in the network and facilitate a better understanding of the molecular mechanisms linked to phenotypes of interest (13). Thus, the present study not only identified differentially expressed genes (DEGs), and DEG-related pathways in PCOS, but also constructed a Reactome function interaction (FI) network based on the interactions between DEGs. Moreover, pathway enrichment analysis was performed for the network modules extracted from the FI network. Furthermore, quantitative RT-PCR was used to detect expression of DEGs which may be important candidate genes in PCOS. The study may shed new light on the molecular mechanisms of PCOS.

\section{Materials and methods}

Preprocessing of microarray data. It was a secondary study of the microarray dataset GSE34526 (9) which was obtained from the Gene Expression Omnibus (GEO) database (14) (http://www.ncbi.nlm.nih. gov/geo/), and based on the Affymetrix Human Genome U133 Plus 2.0 Array platform (15). The microarray dataset consisted of 7 granulosa cell samples from 7 women with PCOS undergoing in vitro fertilization and 3 control granulosa cell samples from 3 normal women undergoing in vitro fertilization. For data preprocessing, the probe-level data in CEL files were converted into expression measures by using the affy package in R language (16), and then was subjected to background correction and quartile data normalization by using robust multiarray average (RMA) algorithm. Each probe was mapped to its corresponding gene using Biconductor annotation function (17) of R language. The probes corresponding to no gene or more than one gene were deleted. When there were several probes for one gene, the averaged expression value of these probes was used as the expression value of the gene. The standardized expression value is shown in a box figure (Fig. 1). It was depicted that the median gene expression value of normal samples is as high as that of PCOS samples, suggesting a marked degree of standardization of the data after preprocessing.

Determination and hierarchical clustering analysis of DEGs. Linear Models for Microarray Analysis package in $\mathrm{R}$ language (18) was employed to screen DEGs between PCOS samples and control normal samples. The strict thresholds were set at fold-change $\left(\log _{2} \mathrm{FCl}\right) \geq 1$ and $\mathrm{P}$-value $<0.05$. The screened DEGs underwent two-way hierarchical clustering analysis by using the pheatmap package (19) in R language (http://cran.fhcrc.org/web/packages/ pheatmap/ index. html).

Pathway enrichment analysis. In order to unveil the pathways that may be associated with the identified DEGs, Kyoto Encyclopedia of Genes and Genomes (KEGG) pathway enrichment analysis was performed using ClueGO plug-in and CluePedia plugin of Cytoscape software. ClueGO plug-in (http://www.ici.upmc.fr/cluego/cluegoDownload.shtml) can extrapolate the biological function of large gene lists by identifying significant gene ontology (GO) terms and KEGG pathways, and functionally categorize the GO terms and KEGG pathways (20). The CluePedia plugin (http://www.ici. upmc.fr/cluepedia/) is used to search for pathway-associated markers and can offer an extensive view of a pathway by studying experimental information and in silico data (21). In this study, a right-side hypergeometric test was used for calculation of the P-value, followed by the multiple test correction [Benjamini-Hochberg adjustment (22)]. A pathway with adjusted P-value $<0.05$ was considered significant.

Based on the Kappa score threshold $(\geq 0.4)$, these significant pathways were functionally divided into several groups and exhibited in a network, in which a node represented a KEGG pathway, and an edge between two nodes indicated that the two pathways shared common genes. Significant KEGG pathways enriched with the DEGs in the present study were visualized using R/Bioconductor package pathview which is a useful tool to map user data onto relevant pathways and offers graphs of the pathways mapped by the user data (23).

Construction of a Reactome FI network and analysis of the network modules. It has been shown that complicated disease phenotypes are better related to changes in networks, instead of a single gene or gene product (24). Therefore, a Reactome FI network was constructed with the DEGs by using ReactomeFIViz. ReactomeFIViz (http://wiki.reactome.org. sci-hub.org/index.php/Reactome_FI_Cytoscape_Plugin) is a Cytoscape app which can construct a Reactome FI network for pathway and network-based analysis of high throughout experimental data, and extract pathway and network patterns associated with diseases (13). In the network, nodes represent genes. An edge between two nodes stands for the interaction between two genes in the network. The edge weight corresponds to the Pearson's correlation coefficient between the two genes.

Network clustering analysis was then performed for the Reactome FI network by using Markov clustering algorithm (25), and highly connected network modules (min module size $\geq 7$; average correlation $\geq 0.25$ ) were extracted from the network. The hub gene in a module referred to the gene that had the most interactions. In order to identify association of the network modules with sample phenotype, the Pearson' correlation coefficient of the genes in each module to the sample phenotype (normal and PCOS samples) was calculated. The mean Pearson's correlation coefficient of all genes included in each module was regarded as the module significance. Moreover, pathway enrichment analysis was also performed for genes in each module using the reactomeFIVZ $(\mathrm{P}<0.05)$ based on the following publicly available databases: Reactome (R) database (26), KEGG (K), National Cancer Institute Pathway Interaction Database (NCI-PID) (N) (27), BioCarta in NCI-PID (B), and PantherDB (P). (28)

Quantitative RT-PCR. Expression of identified DEGs in granulosa cell samples was tested using quantitative RT-PCR. A total of 12 follicular fluid samples were collected for the experiment, including 5 samples from 5 normal women and 7 samples from 7 patients with PCOS, who were undergoing in vitro fertilization. Demographic and clinical characteristics of each subject were collected from medical records, including age, follicle-stimulating hormone (FSH), luteinizing hormone (LH), prolactin (PRL), estradiol (E2), thyroid $(\mathrm{T})$, fasting blood glucose $(\mathrm{FBG})$, fasting plasma 


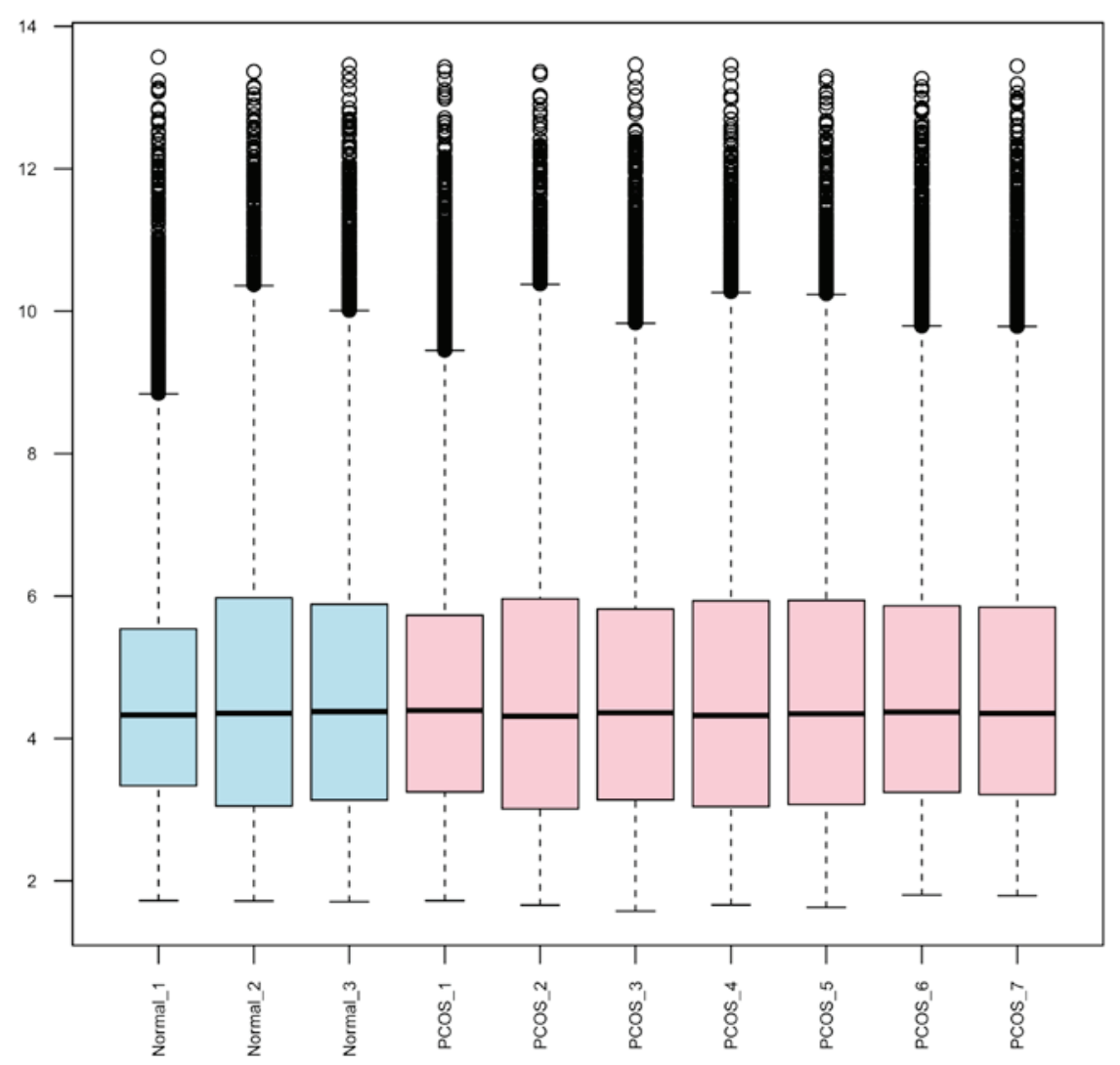

Figure 1. A boxplot of the gene expression profile across samples after preprocessing. Horizontal axis represents sample names; vertical axis represents gene expression value. Blue box stands for normal sample; pink box stands for polycystic ovary syndrome (PCOS) sample. Black horizontal line residing in the box stands for the median of the sample expression value. It shows that the median expression value of normal samples is as high as that of PCOS samples.

Table I. Primers used for the RT-PCR experiment in the study.

\begin{tabular}{ll}
\hline Gene & \multicolumn{1}{c}{ Primer sequences } \\
\hline MYH9 & F: GCCAAGACCGTGAAGAAT \\
& R: CCAGACAGGAGATAATAGAAGA \\
LYN & F: TGAAGCCAGGAACTATGTC \\
& R: TGTACTCGGTGATGATGTAA \\
ARHGAP4 & F: GGATGAGGTGGCTGAGAT \\
& R: GCTGGTCTGGAAGGAATC \\
ARHGAP9 & F: GGACGCTGCTTCTACATAA \\
& R: GACATCATTGTTCCTCTTCAG \\
ACTB & F: TCATGAAGTGTGACGTGGACATC \\
& R: CAGGAGGAGCAATGATCTTGATCT \\
RHOG & F: CTGCTCATCTGCTACACAA \\
& R: CCACAGGTTCAGGTTCAC \\
GAPDH & F: TGACAACTTTGGTATCGTGGAAGG \\
& R: AGGCAGGGATGATGTTCTGGAGAG
\end{tabular}

F, forward; R, reverse.

insulin (FINS), homeostasis model assessment of insulin resistance (HOMA-IR) levels, height, body weight, body mass index (BMI), and number of antral follicles (left and right).
The experiment was approved by the Ethics Committee of Obstetrics and Gynecology Hospital of Fudan University and informed consent was obtained before the use of the samples. Granulosa cells were extracted from the follicular fluid using TRIzol reagent (Invitrogen, Carlsbad, CA, USA). cDNA was synthetized as recommended by the manufacturer using a reverse transcription kit (Takara, Tokyo, Japan). Thermal cycle profiles used in this study were: denaturing for $10 \mathrm{~min}$ at $95^{\circ} \mathrm{C}$, annealing for $15 \mathrm{sec}$ at $95^{\circ} \mathrm{C}$, and extension for $10 \mathrm{sec}$ at $72^{\circ} \mathrm{C}$. PCR was carried out for 30 cycles. Glyceraldehyde-3phosphate dehydrogenase (GAPDH) was used as an internal reference. The set of primers used in the study is shown in Table I.

Statistical analysis. SPSS version 19.0 (IBM Corp., Armonk, NY, USA) was used for data analysis. Continuous variables between two groups were compared using Student's t-test. ANOVA was used to analyze the differences between 3 groups, followed by pairwise comparison using least significant difference test. Differences with P-value $<0.05$ were considered significant.

\section{Results}

Identification and hierarchical clustering analysis of DEGs. Between PCOS and control samples, 674 DEGs were screened, including 506 upregulated genes and 168 downregulated genes. The result of the hierarchical clustering analysis of the DEGs is exhibited in a heatmap plot (Fig. 2). The majority of 

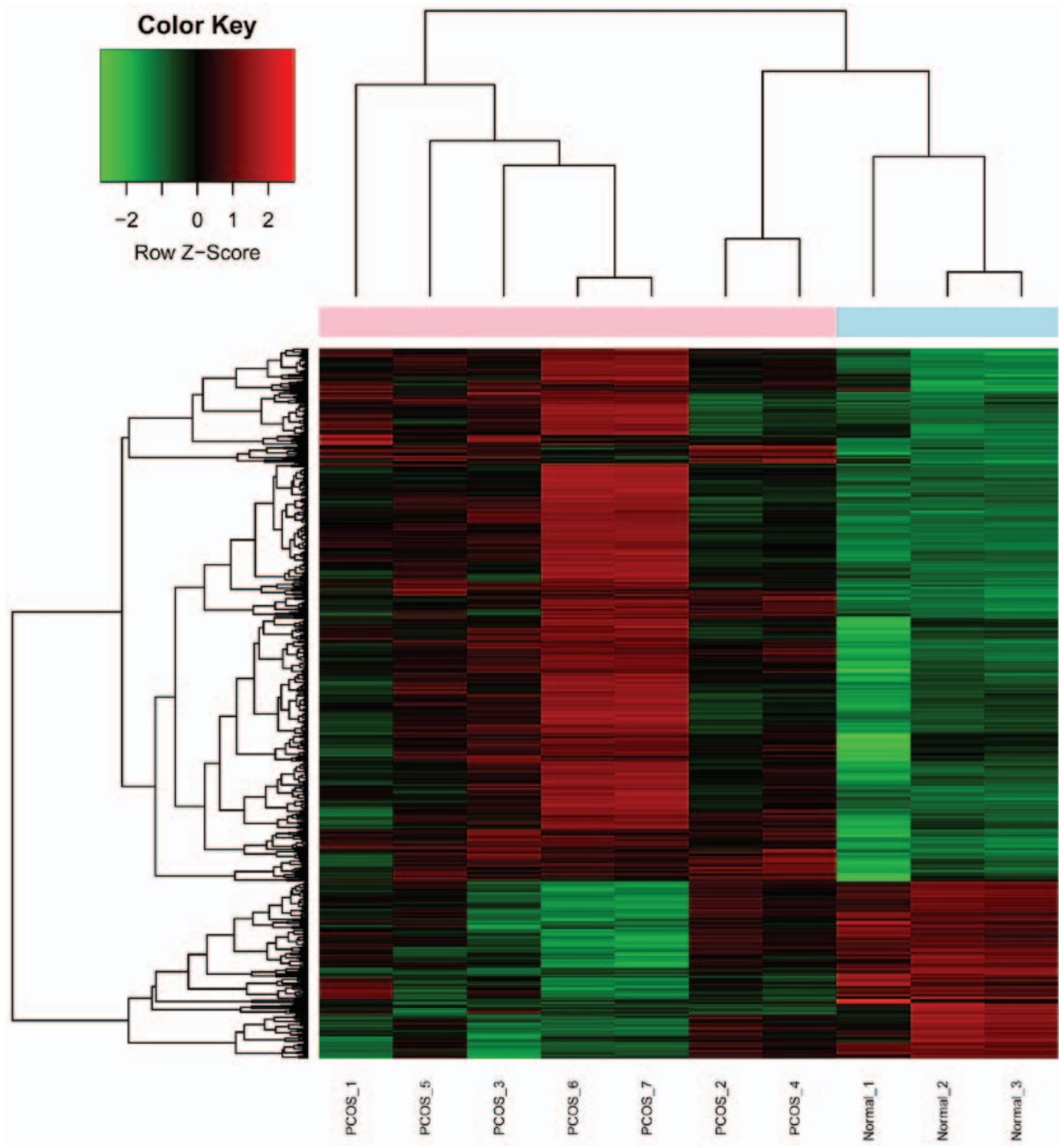

Figure 2. A heatmap plot of the differentially expressed genes (DEGs) of all samples. Horizontal axis represents sample name; vertical axis represents the fold-change of DEG expression. The red denotes expression of upregulated DEGs; the green denotes expression of downregulated DEGs. Color bar above indicates sample type: pink bar for polycystic ovary syndrome (PCOS) samples and blue bar for normal samples.

genes in the PCOS samples was upregulated as compared to those in the control samples.

Pathway enrichment analysis. KEGG pathway enrichment analysis was performed for the DEGs with a view to determining which pathways possibly involved the obtained DEGs. Collectively, 41 pathways were significantly enriched with the DEGs. As shown in Fig. 3, 30 pathways were functionally categorized into 5 groups based on the Kappa score $(\geq 0.4)$. The other 11 pathways were not functionally related to other pathways. These significant pathways were primarily associated with immune and inflammation. Each significant pathway was visualized by the Pathview software. One example was the Cytokine-cytokine receptor interaction pathway (adjusted $\mathrm{P}=1.67 \mathrm{E}^{-05}$ ) (Fig. 4) which was enriched with 31 genes, such as chemokine (C-X-C motif) ligand (CXCL) subfamily members (CXCL3, CXCL6, CXCL10, CXCL16 and CXCL4) and chemokine (C-C motif) ligand (CCL) subfamily members (CCL2, CCL8, CCR5, CCR7 and CCR1).
Analysis of the Reactome FI network and the network modules. For the purpose of evaluating the associations between the identified DEGs, a Reactome FI network was constructed with the DEGs, and 8 highly connected network nodules were extracted from the network (Table II). Fig. 5 shows that among the 8 modules, module 0 had the largest size, including 13 genes with hub gene RAS-related $\mathrm{C} 3$ botulinum substrate 2 (RAC2). Both module 1 and 2 had 11 genes. The hub genes were actin- $\beta$ (ACTB) and $\mathrm{Fc}$ fragment of IgG, low affinity IIc, receptor for (CD32) (FCGR2C), respectively. Additionally, 10 genes were included in module 3 with LYN proto-oncogene, Src family tyrosine kinase (LYN) as the hub genes.

The module significance to sample phenotype (normal and PCOS samples) was calculated to explore the degree of the association of each module with sample phenotype. As shown in Fig. 6, the module significance ranged from 0.702 to 0.81 . This suggested that these modules were well associated with the sample phenotype. Module 3 had the highest module significance (0.81). 


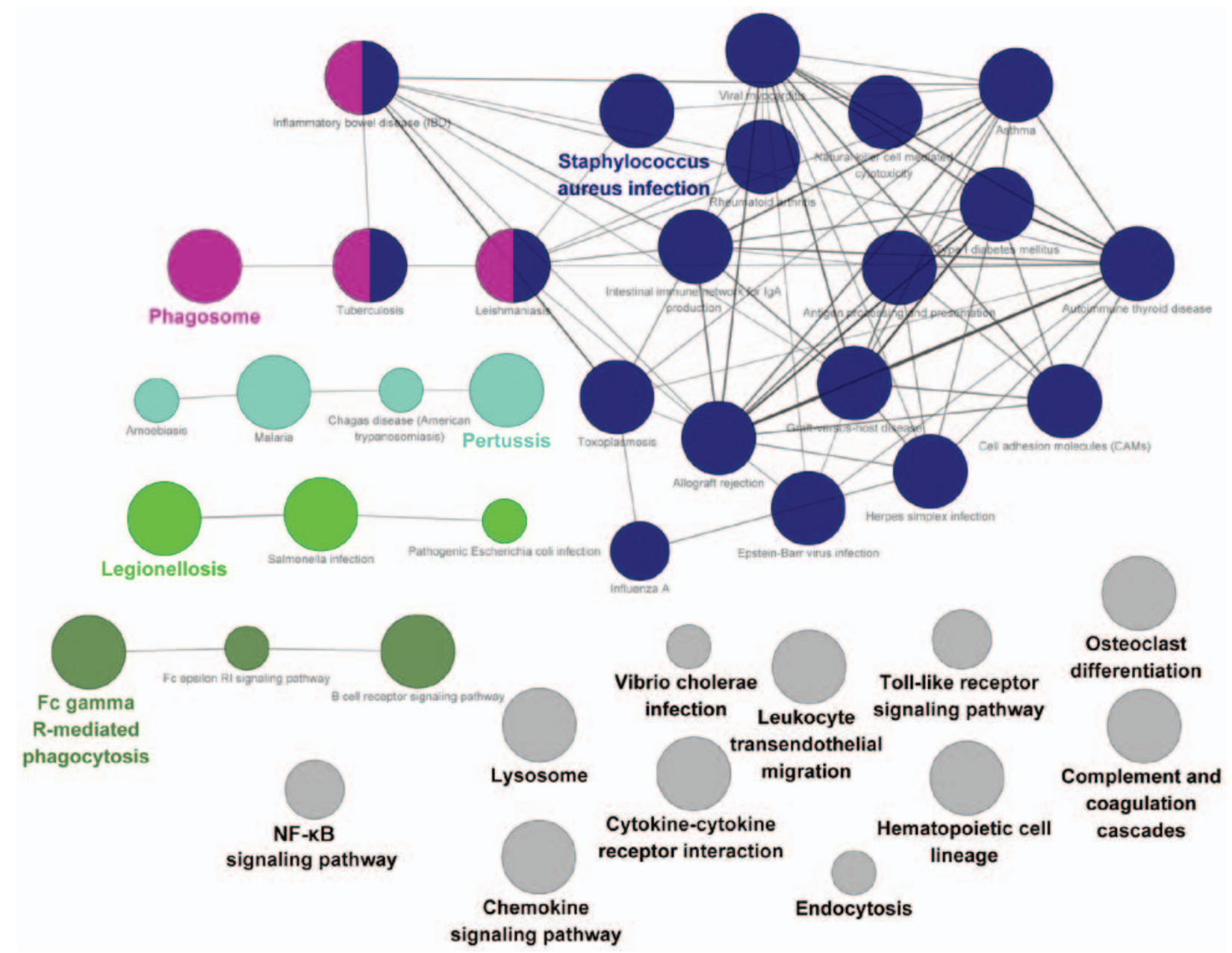

Figure 3. Grouping of significant Kyoto Encyclopedia of Genes and Genomes (KEGG) pathways of the differentially expressed genes (DEGs). A node represents a KEGG pathway, and the node size is negatively correlated with the adjusted P-value of the pathway. An edge between two nodes denotes that the two pathways share common genes. The edge width is positively correlated with the number of common genes. Significant KEGG pathways of DEGs are classified into several functional groups based on $\kappa$ value. Nodes of a group are labeled in the same color. Grey nodes do not share genes with other pathways. The nodes of two colors are shared by two groups.

Table II. Components of 8 network modules.

\begin{tabular}{|c|c|c|c|}
\hline Module & No of genes & $\begin{array}{l}\text { Average } \\
\text { correlation }\end{array}$ & Gene list \\
\hline Module 0 & 13 & 0.8196 & $\begin{array}{l}\text { ARAP1, ARHGAP26, ARHGAP4, ARHGAP9, CORO1 A, HMHA1, NCKAP1L, } \\
\text { PIP5K1B, PKN1, PREX1, RAC2, RHOG, STARD8 }\end{array}$ \\
\hline Module 1 & 11 & 0.8695 & $\begin{array}{l}\text { ACTB, ARPC1B, CFL2, COL8A2, EPB41L3, MYH9, MYO1G, P2RX7, PARVG, } \\
\text { PSTPIP1, ZYX }\end{array}$ \\
\hline Module 2 & 11 & 0.8501 & $\begin{array}{l}\text { BLNK, CD72, CLEC7A, FCGR2B, FCGR2C, LILRA2, LILRA5, LILRA6, } \\
\text { OSCAR, SLA, SYK }\end{array}$ \\
\hline Module 3 & 10 & 0.8006 & CD24, CD53, DAB2, FCER1G, FGR, HCK, LPAR3, LYN, SHC4, TNFRSF11A \\
\hline Module 4 & 8 & 0.8748 & COL15A1, FERMT3, FLNA, ICAM3, ITGB2, RASSF5, SELPLG, TGFBI \\
\hline Module 5 & 8 & 0.7448 & CXCL16, CXCL3, CXCL6, GNAI2, NPY2R, NUCB1, P2RY13, SUCNR1 \\
\hline Module 6 & 8 & 0.6387 & $\begin{array}{l}\text { AP1S2, HLA-DMA, HLA-DPA1, HLA-DQA1, HLA-DQB1, HLA-DRA, } \\
\text { LGMN, RACGAP1 }\end{array}$ \\
\hline Module 7 & 7 & 0.6262 & CD14, CD180, LY86, LY96, TLR1, TLR4, TNFRSF1B \\
\hline
\end{tabular}

Average correlation: average absolute edge weight of edges in a module. 

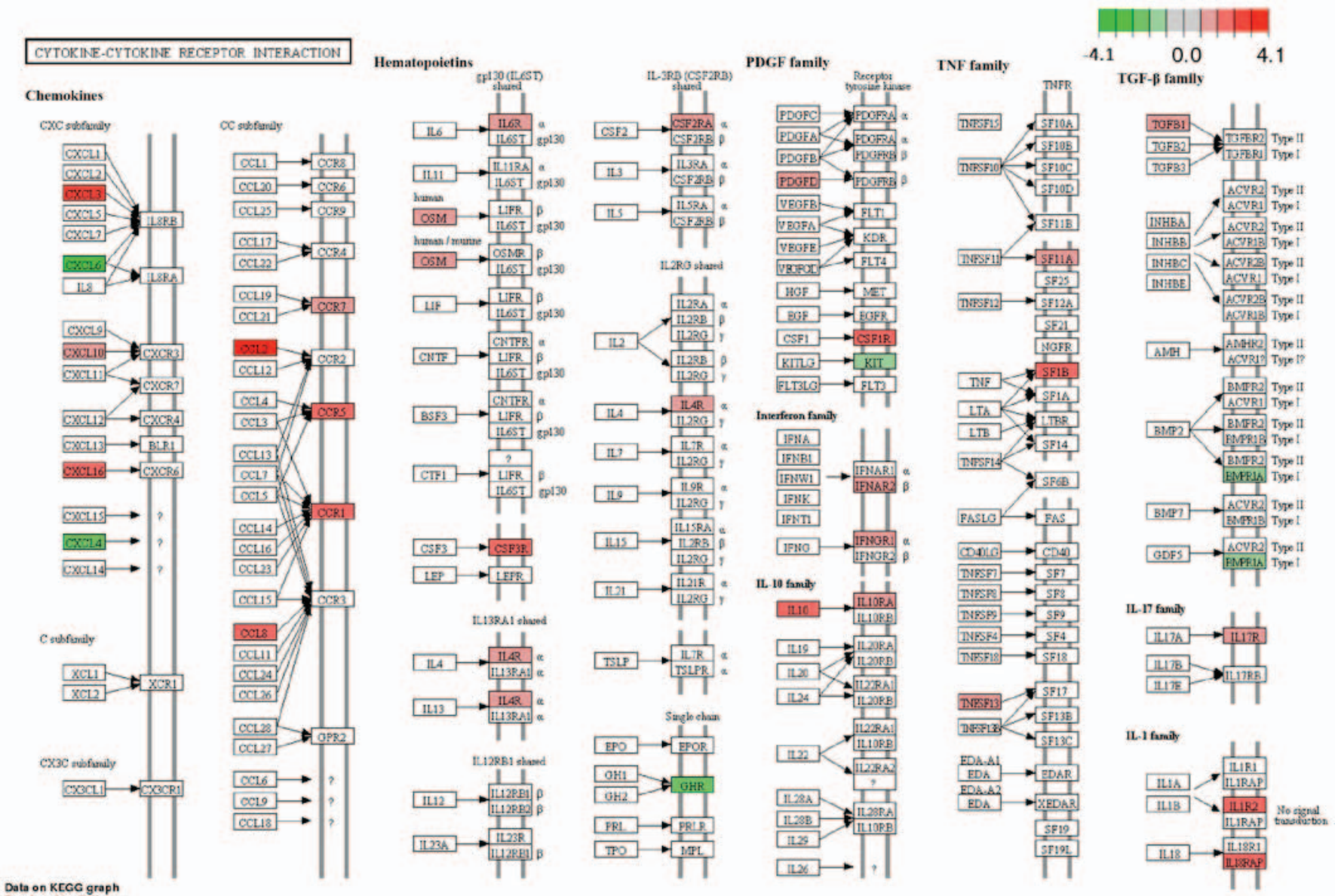

Figure 4. Pathview of the Cytokine-cytokine receptor interaction pathway. Node color change reflects log fold-change (FC) range.

Pathway enrichment analysis was also performed for the genes in each module. Top 5 significant pathways for each network module were identified based on the P-value (Table III). Module 0 was significantly enriched with the Regulation of RhoA activity, Signaling by Rho GTPases, Regulation of RAC1 activity, GPVI-mediated activation cascade and RhoA signaling pathways. Rho GTPase activating protein (ARHGAP)4 and ARHGAP9 are involved in the Regulation of RhoA activity and Signaling by Rho GTPase pathways. In addition, Ras homolog family member G (RHOG) was related to Signaling by Rho GTPases and GlycoProtein (GP)VI-mediated activation cascade pathways. Module 1 was associated with the Regulation of actin cytoskeleton, Salmonella infection, Nicotinic acetylcholine receptor signaling, and Tight junction pathways. ACTB and myosin, heavy chain 9, non-muscle (MYH9) were enriched in the 4 pathways. Module 2 was related to the Tuberculosis, Osteoclast differentiation, B cell receptor signaling, BCR signaling and Phagosome signaling pathways. Module 3 was related to Thromboxane A2 receptor signaling, Signaling events mediated by PTP1B, Chemokine signaling, CXCR4-mediated signaling events and ephrin B reverse signaling pathways. LYN, hub gene of module 3 was enriched in each of these pathways. Notably, module 6 was related to MHC class II antigen presentation pathway which was enriched with several human leukocyte antigen (HLA) genes, such as HLA-DQB1, HLA-DPA1, HLA-DMA, HLA-DQA1 and HLA-DRA. Module 7 was linked to Toll-like receptorrelated pathways.

RT-PCR analysis. Bioinformatic analysis showed that ARHGAP4, ARHGAP9, RHOG, ACTB, MYH9 and LYN were upregulated DEGs in PCOS samples relative to normal samples. In order to verify these results, quantitative RT-PCR was used to measure the mRNA expression of these genes in granulosa cell samples of PCOS patients and normal controls. With regard to demographic and clinical characteristics (Table IV), PCOS patients and normal controls were not significantly different in regards to age, FSH, LH, PRL, E2, T and height. Yet, PCOS patients had markedly increased FBG, FINS, HOMA-IR, body weight, BMI and number of antral follicles. As shown in Fig. 7, ARHGAP4, ARHGAP9, RHOG and LYN at the mRNA level were significantly increased in PCOS compared to levels in the normal controls $(\mathrm{P}<0.05)$. Although increased mRNA expression of ACTB and MYH9 was also observed in PCOS, the difference was not significant $(\mathrm{P}>0.05)$.

Furthermore, the 7 PCOS samples were divided into two groups: PCOS non-IR $(n=3)$ and PCOS IR $(n=4)$ groups. The demographic and clinical characteristic analysis revealed that LH and E2 were significantly decreased, and FINS, HOMA-IR and BMI were significantly increased in PCOS IR patients relative to PCOS non-IR patients (Table V). Results of RT-PCR found that the PCOS IR group had markedly 


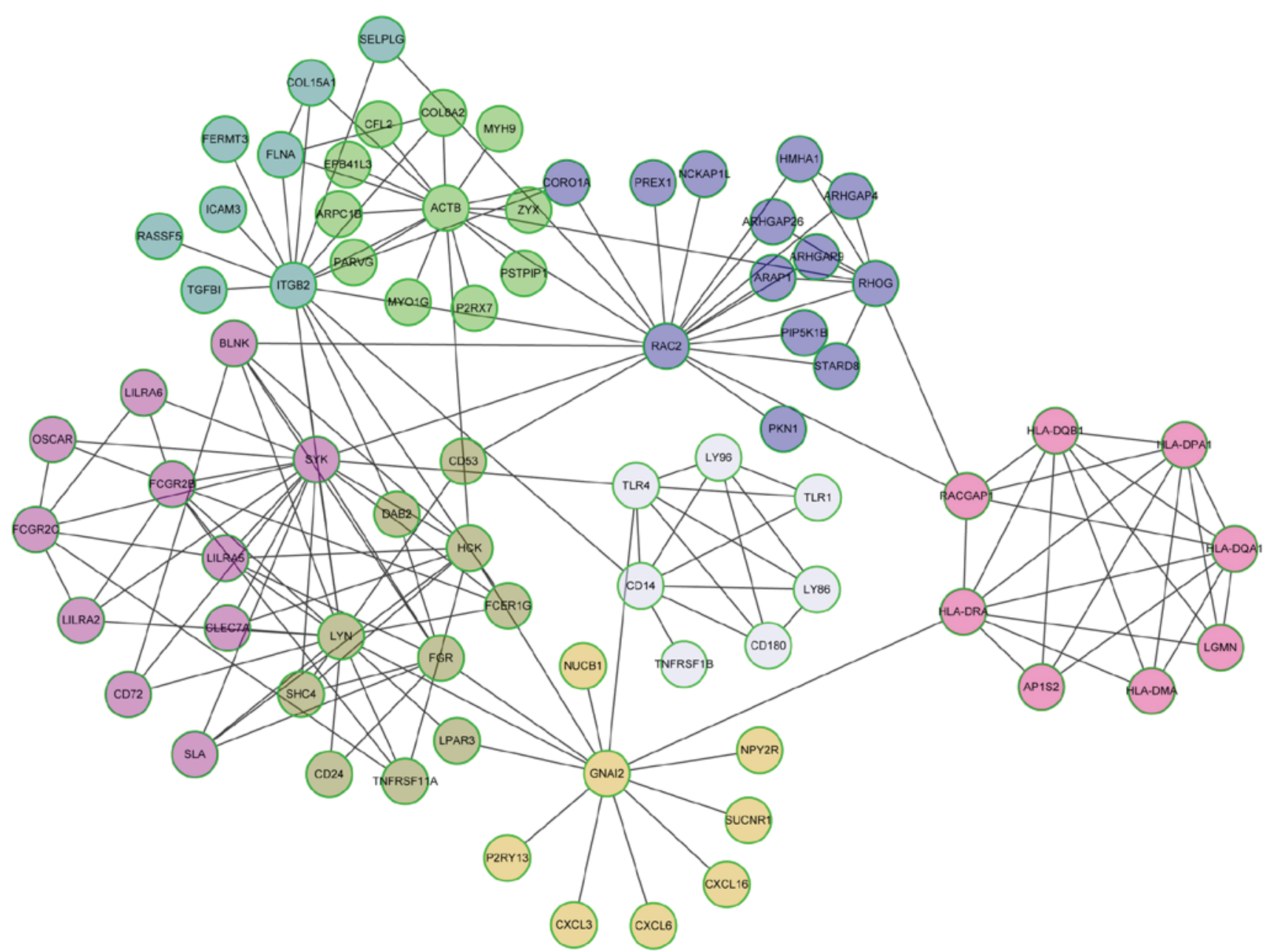

Figure 5. Eight network modules extracted from the Reactome function interaction network. A node stands for a differentially expressed gene (DEG); the link between two nodes reflects the interaction of the two DEGs. Nodes of one module are shown in the same color. The node that has the most links in a module is defined as the hub gene of the module.

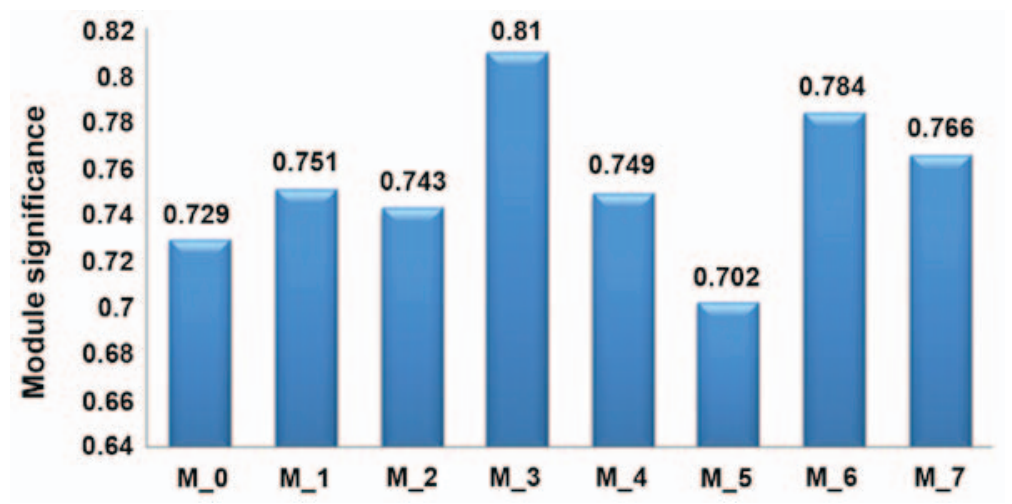

Figure 6. Module significance. Horizontal axis indicates module name; vertical axis indicates module significance. The mean Pearson's correlation coefficient of all genes in each module to sample phenotype is defined as module significance.

elevated expression of ARHGAP4, ARHGAP9, RHOG and LYN when compared to these parameters in the PCOS non-IR group (Fig. 8).

\section{Discussion}

PCOS is a complex disease characterized by variable clinical features. It is largely ascribed to hormone imbalance and results in a heavy health and economic burden. The present study identified a list of genes and pathways that may be involved in the pathogenesis of PCOS by a series of microarray analyses. A total of 674 DEGs were screened between PCOS and control samples. Pathway enrichment analysis revealed significant enrichment of 41 pathways with the DEGs. These pathways were predominately related to immune and inflammation. In line with the finding, it has been reported that inflammation is 
Table III. Top 5 significant KEGG pathways for each network module.

\begin{tabular}{|c|c|c|c|c|}
\hline Module & Pathway & No. of genes. & P-value & Gene list \\
\hline Module 0 & Regulation of RhoA activity & 3 & 0 & ARHGAP4, ARAP1, ARHGAP9 \\
\hline Module 0 & Signaling by Rho GTPases & 8 & 0 & $\begin{array}{l}\text { ARHGAP26, ARHGAP4, HMHA1, RAC2, } \\
\text { STARD8, ARAP1, RHOG, ARHGAP9 }\end{array}$ \\
\hline Module 0 & Regulation of RAC1 activity & 2 & 0.0012 & PREX1, ARHGAP9 \\
\hline Module 0 & GPVI-mediated activation cascade & 2 & 0.0015 & RAC2, RHOG \\
\hline Module 0 & RhoA signaling pathway & 2 & 0.0016 & PIP5K1B, PKN1 \\
\hline Module 1 & Regulation of actin cytoskeleton & 4 & 0 & ACTB, ARPC1B, CFL2, MYH9 \\
\hline Module 1 & Salmonella infection & 3 & 0.0001 & ACTB, ARPC1B, MYH9 \\
\hline Module 1 & $\begin{array}{l}\text { Nicotinic acetylcholine receptor } \\
\text { signaling pathway }\end{array}$ & 2 & 0.0002 & АCTB, MYH9 \\
\hline Module 1 & Tight junction & 3 & 0.0003 & ACTB, EPB41L3, MYH9 \\
\hline Module 1 & Integrin signaling pathway & 3 & 0.0005 & ACTB, ARPC1B, COL8A2 \\
\hline Module 2 & Tuberculosis & 4 & 0 & FCGR2B, FCGR2C, CLEC7A, SYK \\
\hline Module 2 & Osteoclast differentiation & 8 & 0 & $\begin{array}{l}\text { FCGR2B, LILRA2, FCGR2C, OSCAR, LILRA5, } \\
\text { LILRA6, BLNK, SYK }\end{array}$ \\
\hline Module 2 & B cell receptor signaling pathway & 4 & 0 & FCGR2B, CD72, BLNK, SYK \\
\hline Module 2 & BCR signaling pathway & 4 & 0 & FCGR2B, CD72, BLNK, SYK \\
\hline Module 2 & Phagosome & 3 & 0.0006 & FCGR2B, FCGR2C, CLEC7A \\
\hline Module 3 & Thromboxane A2 receptor signaling & 3 & 0 & FGR, LYN, HCK \\
\hline Module 3 & Signaling events mediated by РТР1B & 3 & 0 & FGR, LYN, HCK \\
\hline Module 3 & Chemokine signaling pathway & 4 & 0 & FGR, LYN, HCK, SHC4 \\
\hline Module 3 & CXCR4-mediated signaling events & 3 & 0 & FGR, LYN, HCK \\
\hline Module 3 & Ephrin B reverse signaling & 3 & 0 & FGR, LYN, HCK \\
\hline Module 4 & $\beta 2$ integrin cell surface interactions & 3 & 0 & ICAM3, TGFBI, ITGB2 \\
\hline Module 4 & Cell adhesion molecules (CAMs) & 3 & 0.0002 & ICAM3, ITGB2, SELPLG \\
\hline Module 4 & Integrin signaling pathway & 3 & 0.0002 & COL15A1, ITGB2, FLNA \\
\hline Module 4 & amb2 integrin signaling & 2 & 0.0003 & ITGB2, SELPLG \\
\hline Module 4 & Extracellular matrix organization & 3 & 0.0008 & ICAM3, COL15A1, ITGB2 \\
\hline Module 5 & GPCR ligand binding & 6 & 0 & $\begin{array}{l}\text { P2RY13, CXCL16, CXCL3, NPY2R, } \\
\text { SUCNR1, CXCL6 }\end{array}$ \\
\hline Module 5 & Chemokine signaling pathway & 4 & 0 & GNAI2, CXCL16, CXCL3, CXCL6 \\
\hline Module 5 & GPCR downstream signaling & 7 & 0 & $\begin{array}{l}\text { P2RY13, GNAI2, CXCL16, CXCL3, NPY2R, } \\
\text { SUCNR1, CXCL6 }\end{array}$ \\
\hline Module 5 & Cytokine-cytokine receptor interaction & 3 & 0.0006 & CXCL16, CXCL3, CXCL6 \\
\hline Module 5 & Pertussis & 2 & 0.0012 & GNAI2, CXCL6 \\
\hline Module 6 & Epstein-Barr virus infection & 4 & 0 & HLA-DQB1, HLA-DPA1, HLA-DQA1, HLA-DRA \\
\hline Module 6 & MHC class II antigen presentation & 8 & 0 & $\begin{array}{l}\text { HLA-DQB1, AP1S2, LGMN, HLA-DPA1, } \\
\text { RACGAP1, HLA-DMA, HLA-DQA1, HLA-DRA }\end{array}$ \\
\hline Module 6 & Viral myocarditis & 5 & 0 & $\begin{array}{l}\text { HLA-DQB1, HLA-DPA1, HLA-DMA, } \\
\text { HLA-DQA1, HLA-DRA }\end{array}$ \\
\hline Module 6 & Staphylococcus aureus infection & 5 & 0 & $\begin{array}{l}\text { HLA-DQB1, HLA-DPA1, HLA-DMA, } \\
\text { HLA-DQA1, HLA-DRA }\end{array}$ \\
\hline Module 6 & Autoimmune thyroid disease & 5 & 0 & $\begin{array}{l}\text { HLA-DQB1, HLA-DPA1, HLA-DMA, } \\
\text { HLA-DQA1, HLA-DRA }\end{array}$ \\
\hline Module 7 & Pertussis & 3 & 0 & LY96, TLR4, CD14 \\
\hline Module 7 & $\mathrm{NF}-\kappa \mathrm{B}$ signaling pathway & 3 & 0 & LY96, TLR4, CD14 \\
\hline Module 7 & Toll-like receptors cascades & 6 & 0 & LY96, LY86, TLR1, TLR4, CD14, CD180 \\
\hline Module 7 & Pathogenic Escherichia coli infection & 3 & 0 & LY96, TLR4, CD14 \\
\hline Module 7 & Toll-like receptor signaling pathway & 4 & 0 & LY96, TLR1, TLR4, CD14 \\
\hline
\end{tabular}

KEGG, Kyoto Encyclopedia of Genes and Genomes. 

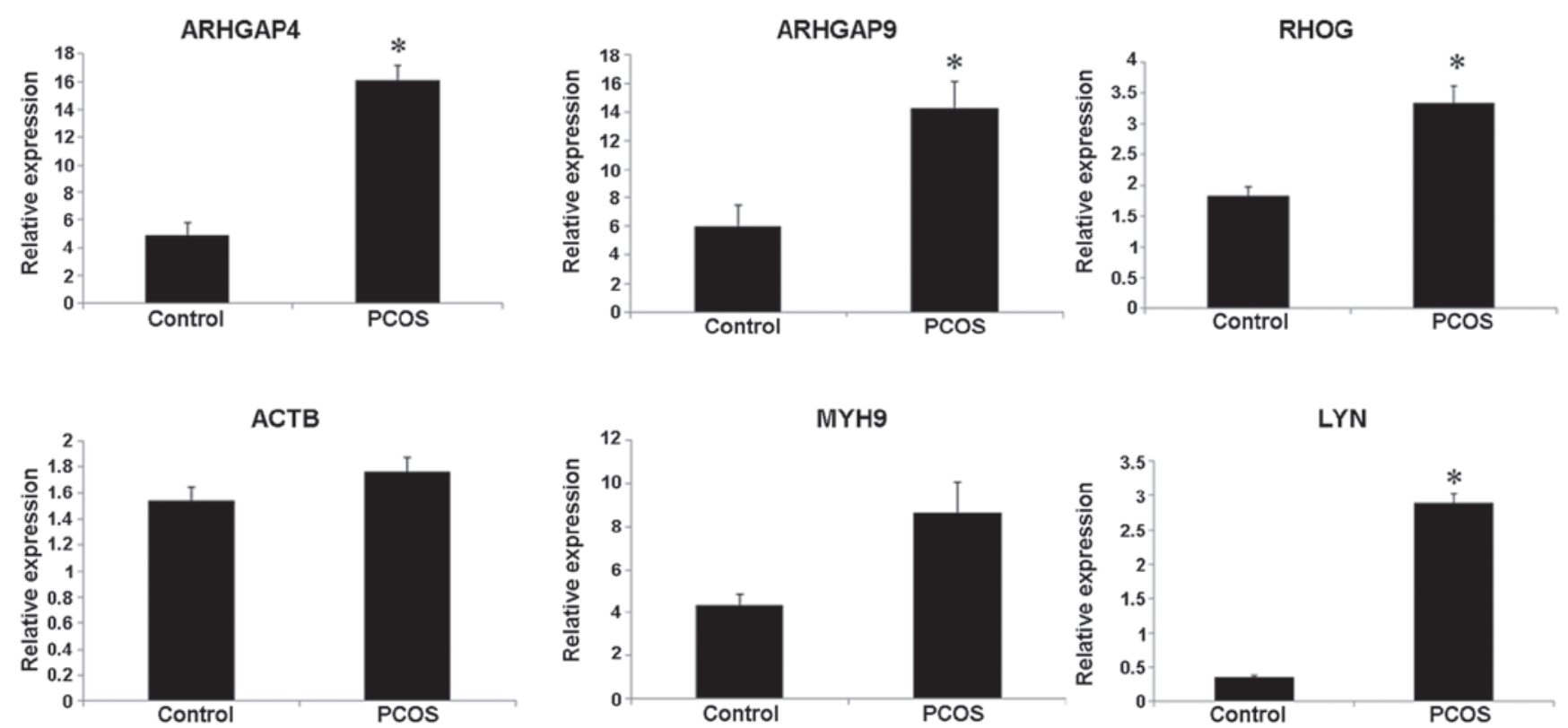

Figure 7. mRNA levels of ARHGAP4, ARHGAP9, RHOG, ACTB, MYH9 and LYN in granulosa cell samples of polycystic ovary syndrome (PCOS) patients and normal controls as detected using RT-PCR. "P<0.05 compared to control. PCOS, polycystic ovary syndrome; ARHGAP, Rho GTPase activating protein; RHOG, Ras homolog family member G; ACTB, actin- $\beta$; MYH9, myosin, heavy chain 9, non-muscle; LYN, LYN proto-oncogene, Src family tyrosine kinase.

Table IV. Demographic and clinical characteristics of controls and PCOS patients for PCR analysis.

\begin{tabular}{lrcr}
\hline Variable & $\begin{array}{c}\text { Control group } \\
(\mathrm{n}=5)\end{array}$ & $\begin{array}{c}\text { PCOS group } \\
(\mathrm{n}=7)\end{array}$ & P-value \\
\hline Age (years) & $27.000 \pm 2.121$ & $28.857 \pm 4.670$ & 0.379 \\
FSH (mIU/ml) & $5.438 \pm 0.514$ & $5.267 \pm 0.404$ & 0.532 \\
LH (mIU/ml) & $4.196 \pm 0.641$ & $4.174 \pm 1.988$ & 0.982 \\
PRL (ng/ml) & $14.988 \pm 2.296$ & $19.986 \pm 4.676$ & 0.054 \\
E2 (pg/ml) & $29.200 \pm 8.044$ & $32.857 \pm 13.031$ & 0.593 \\
T (ng/ml) & $0.268 \pm 0.040$ & $0.294 \pm 0.157$ & 0.725 \\
FBG (mmol/l) & $5.200 \pm 0.158$ & $5.443 \pm 0.288$ & $\mathbf{0 . 0 9 2}$ \\
FINS (mIU/l) & $5.980 \pm 0.858$ & $10.829 \pm 2.780$ & $\mathbf{0 . 0 0 4}$ \\
HOMA-IR & $1.380 \pm 0.191$ & $2.637 \pm 0.759$ & $\mathbf{0 . 0 0 5}$ \\
Height (m) & $1.584 \pm 0.054$ & $1.607 \pm 0.049$ & 0.466 \\
Body weight (kg) & $54.000 \pm 8.602$ & $70.286 \pm 9.656$ & $\mathbf{0 . 0 1 3}$ \\
BMI & $21.467 \pm 2.704$ & $27.204 \pm 3.372$ & $\mathbf{0 . 0 1 1}$ \\
Number of antral & $6.800 \pm 1.304$ & $21.714 \pm 5.851$ & $<\mathbf{0 . 0 0 1}$ \\
follicles (L) & & & \\
Number of antral & $7.800 \pm 1.304$ & $25.714 \pm 2.870$ & $<\mathbf{0 . 0 0 1}$ \\
follicles (R) & & & \\
\hline
\end{tabular}

Differences between two groups were analyzed using Student's t-test. P-values marked in bold are $<0.05$. PCOS, polycystic ovary syndrome; FSH, follicle-stimulating hormone; $\mathrm{LH}$, luteinizing hormone; PRL, prolactin; E2, estradiol; T, thyroid; FBG, fasting blood glucose; FINS, fasting plasma insulin; HOMA-IR, homeostasis model assessment of insulin resistance; BMI, body mass index.

enhanced, and production of interleukins and chemokines is increased in PCOS $(29,30)$. Our result confirms the crucial role of inflammation and immune in the development of PCOS.
In the present study, 8 highly connected network modules were extracted from the Reactome FI network. Among these network modules, module 3 had the highest module significance. LYN was the hub gene of module 3. Notably, microarray analysis revealed that LYN was an upregulated DEG in PCOS, which was in accordance with the result of RT-PCR. LYN encodes tyrosine-protein Lyn which is a member of the src family tyrosine kinase. There is in vivo evidence that Src family kinases are essential for generation of the inflammatory environment (31). Furthermore, research has established that Lyn plays both a positive and a negative regulatory role in neutrophils and macrophages (32). Lyn plays a prominent role in the activation of innate immune response mediated by nuclear factor- $\kappa \mathrm{B}$ in human mononuclear cells (33). These findings suggest that Lyn is closely related to inflammation and immune response. In the present study, pathway analysis for module 3 revealed that LYN was significantly enriched in the Inflammation and immune-related TXA2 receptor signaling, Chemokine signaling, and CXCR4-mediated signaling events pathways, which was in concordance with previous findings. Dysregulated LYN may participate in PCOS-related inflammation and immune response. Furthermore, inflammation is an important mechanism underlying insulin resistance (34), which is prevalent in PCOS patients (35). By using RT-PCR, the study found that LYN expression was significantly increased in PCOS IR patients compared with PCOS non-IR patients. This indicates that upregulated LYN may be associated with PCOS-related insulin resistance as well.

Pathway enrichment analysis revealed that module 0 was significantly associated with Regulation of RhoA activity, Signaling by Rho GTPases and RhoA signaling pathways. RhoA is a member of Rho family of GTPases involved in regulating intracellular actin dynamics (36). It has been demonstrated that RhoA participates in regulating secretion of insulin (37), and is associated with insulin resistance via phos- 
Table V. Demographic and clinical characteristics of controls, PCOS non-IR patients and PCOS IR patients for PCR analysis.

\begin{tabular}{lcccrr}
\hline Variable & $\begin{array}{c}\text { Control group } \\
(\mathrm{n}=5)\end{array}$ & $\begin{array}{c}\text { PCOS non-IR group } \\
(\mathrm{n}=3)\end{array}$ & $\begin{array}{c}\text { PCOS IR group } \\
(\mathrm{n}=4)\end{array}$ & F-value & P-value \\
\hline Age (years) & $27.000 \pm 2.121$ & $30.000 \pm 5.657$ & $28.400 \pm 4.879$ & 0.425 & 0.666 \\
FSH $(\mathrm{mIU} / \mathrm{ml})$ & $5.438 \pm 0.514$ & $5.705 \pm 0.092$ & $5.092 \pm 0.330$ & 1.866 & 0.210 \\
LH $(\mathrm{mIU} / \mathrm{ml})$ & $4.196 \pm 0.641$ & $6.560 \pm 0.750^{\mathrm{a}}$ & $3.220 \pm 1.343^{\mathrm{b}}$ & 7.615 & $\mathbf{0 . 0 1 2}$ \\
PRL $(\mathrm{ng} / \mathrm{ml})$ & $14.988 \pm 2.296$ & $17.260 \pm 1.485$ & $21.076 \pm 5.200$ & 3.206 & 0.089 \\
E2 $(\mathrm{pg} / \mathrm{ml})$ & $29.200 \pm 8.044$ & $50.000 \pm 4.243^{\mathrm{a}}$ & $26.000 \pm 6.671^{\mathrm{b}}$ & 8.528 & $\mathbf{0 . 0 0 8}$ \\
T $(\mathrm{ng} / \mathrm{ml})$ & $0.268 \pm 0.040$ & $0.450 \pm 0.212$ & $0.232 \pm 0.093$ & 3.651 & 0.069 \\
FBG $(\mathrm{mmol} / \mathrm{l})$ & $5.200 \pm 0.158$ & $5.200 \pm 0.000$ & $5.540 \pm 0.288$ & 3.512 & 0.075 \\
FINS $(\mathrm{mIU} / \mathrm{l})$ & $5.980 \pm 0.858$ & $7.500 \pm 1.980$ & $12.160 \pm 1.689^{\mathrm{a}, \mathrm{b}}$ & 24.516 & $<\mathbf{0 . 0 0 1}$ \\
HOMA-IR & $1.380 \pm 0.191$ & $1.733 \pm 0.458$ & $2.999 \pm 0.490^{\mathrm{a}, \mathrm{b}}$ & 23.613 & $<\mathbf{0 . 0 0 1}$ \\
Height $(\mathrm{m})$ & $1.584 \pm 0.054$ & $1.635 \pm 0.007$ & $1.596 \pm 0.055$ & 0.709 & 0.517 \\
Body weight $(\mathrm{kg})$ & $54.000 \pm 8.602$ & $61.000 \pm 7.071$ & $74.000 \pm 8.185^{\mathrm{a}}$ & 7.439 & $\mathbf{0 . 0 1 2}$ \\
BMI & $21.467 \pm 2.704$ & $22.808 \pm 2.448$ & $28.962 \pm 1.425^{\mathrm{a}, \mathrm{b}}$ & 15.581 & $\mathbf{0 . 0 0 1}$ \\
Number of antral follicles (L) & $6.800 \pm 1.304$ & $23.000 \pm 9.899^{\mathrm{a}}$ & $21.200 \pm 5.070^{\mathrm{a}}$ & 14.163 & $\mathbf{0 . 0 0 2}$ \\
Number of antral follicles (R) & $7.800 \pm 1.304$ & $25.500 \pm 0.707$ & $25.800 \pm 3.493^{\text {a }}$ & 75.092 & $<\mathbf{0 . 0 0 1}$ \\
\hline
\end{tabular}

ANOVA is applied to analyze the differences between 3 groups, followed by pairwise comparison using least significant difference test. $\mathrm{P}$-values marked in bold are $<0.05$. ${ }^{\mathrm{a}} \mathrm{P}<0.05$ compared to the control group; ${ }^{\mathrm{b}} \mathrm{P}<0.05$ compared to the PCOS non-IR group. PCOS, polycystic ovary syndrome; FSH, follicle-stimulating hormone; LH, luteinizing hormone; PRL, prolactin; E2, estradiol; T, thyroid; FBG, fasting blood glucose; FINS, fasting plasma insulin; HOMA-IR, homeostasis model assessment of insulin resistance; BMI, body mass index.
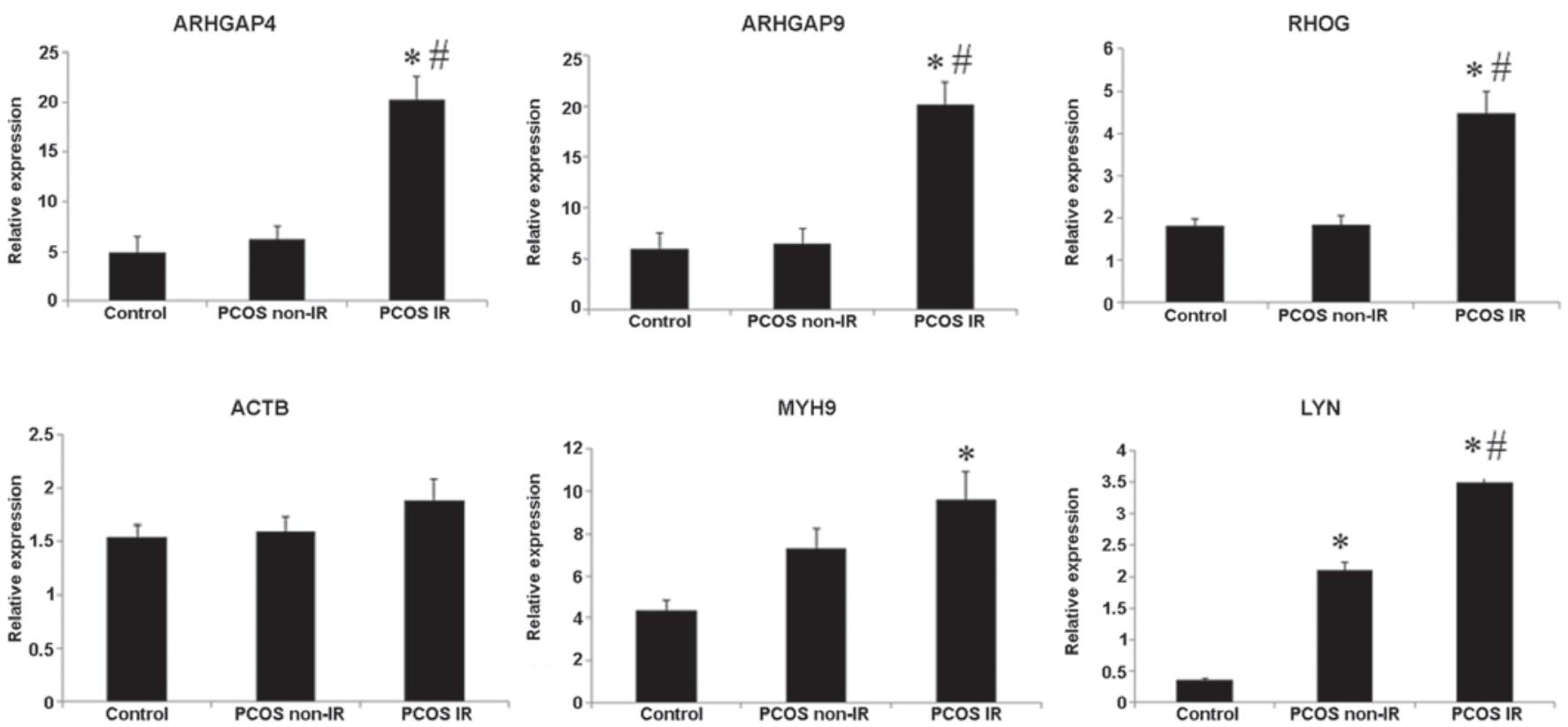

Figure 8. mRNA levels of ARHGAP4, ARHGAP9, RHOG, ACTB, MYH9 and LYN in granulosa cell samples of polycystic ovary syndrome (PCOS) non-IR patients, PCOS IR patients and normal controls as detected using RT-PCR. " $\mathrm{P}<0.05$ compared to the control; ${ }^{*} \mathrm{P}<0.05$ compared to PCOS non-IR patients. IR, insulin, resistance.

phorylation of insulin receptor substrate-1 (38). Moreover, it also mediates adrenocorticotropin-stimulated cortisol biosynthesis in human adrenocortical cells (39). These observations indicate that Regulation of RhoA activity, Signaling by Rho GTPases and RhoA signaling pathways may be associated with PCOS-related hormone imbalance, in particular insulin resistance. Furthermore, the present study also found that Regulation of RhoA activity and Signaling by Rho GTPases pathways shared 2 DEGs: ARHGAP4 and ARHGAP9. They are two members of the Rho-GAP family of GTPase activating proteins participating in the regulation of the function of Rho GTPases (40). Moreover, results of the microarray analysis and RT-PCR reached an agreement that ARHGAP4 and ARHGAP9 were significantly upregulated in PCOS. It has been speculated that dysregulated ARHGAP4 and ARHGAP9 may play a role in PCOS-related hormone imbal- 
ance, particular insulin resistance, by regulating Regulation of RhoA activity and Signaling by Rho GTPases pathways. Consistently, the study found that expression of ARHGAP4 and ARHGAP9 were markedly increased in PCOS IR patients relative to PCOS non-IR patients by using RT-PCR.

RhoG encoded by gene RHOG is a small $G$ protein and belongs to the Rac subfamily of the Rho family. Recently, it has been reported that $\mathrm{RhoG}$ protein may be implicated in glycoprotein VI-Fc receptor $\gamma$-chain complex-mediated platelet activation (41). In agreement with the finding, the study found that RHOG in module 0 was significantly enriched in the GP VI-mediated activation cascade pathway. Furthermore, it has been established that impaired platelet function plays a part in the pathogenesis of PCOS (42). These observations indicate that RHOG and GP VI-mediated activation cascade pathway may be implicated in PCOS-related platelet dysfunction. Moreover, the study found an obviously increased expression of RHOG in PCOS IR patients relative to PCOS non-IR patients. Platelets are important players in inflammation (43), which is a critical mechanism of insulin resistance. Upregulated RHOG may play a role in insulin resistance by affecting platelet dysfunction.

The study found that ACTB and MYH9 in module 1 were upregulated DEGs in PCOS, and significantly related to the Regulation of actin cytoskeleton and Tight junction pathways. These pathways and genes are related to cytoskeleton formation. However, RT-PCR measurement of the two genes revealed that the differences in mRNA expression of ACTB and MYH9 did not reach significance between PCOS and normal samples. This suggests that results of the two genes may be false-positive results of the microarray data analysis. Nevertheless, the abnormal expression of cytoskeletal proteins has been reported in PCOS $(44,45)$. Therefore, more studies are needed to clarify the role of ACTB and MYH9 in PCOS.

HLA genes encode antigen-presenting proteins located on the cell surface in the human body. There is evidence that possession of HLA-DQA1 ${ }^{*} 0501$, HLA-A11 and HLA-DRB1*0403 are risk factors of PCOS $(46,47)$. This study also found that the HLA genes were closely associated with PCOS, as evidenced by the fact that a group of HLA genes (HLA-DQB1, HLA-DPA1, HLA-DMA, HLA-DQA1 and HLA-DRA) were included in module 6 and enriched in MHC class II antigen presentation pathway. Moreover, serum HLA-G in women with PCOS has been reported to be associated with oxidative stress, ovarian hyperandrogenism and insulin resistance (48). It is speculated that HLA-DQB1, HLA-DPA1, HLA-DMA, HLA-DQA1 and HLA-DRA may play a role in PCOS-related oxidative stress, ovarian hyperandrogenism and insulin resistance by affecting the MHC class II antigen presentation pathway. In the present study, genes in module 7 were linked to Toll-like receptorrelated pathways. Toll-like receptors are important participants of the immune system. Toll-like receptor 3 affects $\beta$-cell insulin secretion and glucose homeostasis (49), and activation of Toll-like receptor signaling pathways aid in inducing insulin resistance (50). These findings lead to a speculation that Tolllike receptor signaling pathways are possibly implicated in PCOS-related insulin resistance and immune abnormality.

The study has some limitations. Firstly, its sample size is small. Follicular fluid samples are not easy to collect and many patients may not agree with the study. Secondly, the microarray dataset GSE34526 did not provide clear information on whether the PCOS samples in the dataset were resistant to insulin or not, thus differentially expressed genes between PCOS IR and the PCOS non-IR samples were not distinguished. Thirdly, it is necessary to validate the results of microarray data analysis using experimental methods. In this preliminary study, expression of 6 DEGs in granulosa cell samples of PCOS patients and normal controls were measured using RT-PCR. Further studies with more experiments, such as western blotting, and a large cohort of samples are in process to verify and extend the findings of the present study.

In conclusion, the present study confirmed the important role of inflammation and immune in PCOS. The Regulation of RhoA activity, Signaling by Rho GTPases, and GP VI-mediated activation cascade pathways may be associated with PCOS-related hormone imbalance and platelet dysfunction. LYN, ARHGAP4, ARHGAP9 and RHOG are promising candidate genes in PCOS, and may be recommended as possible therapeutic targets for PCOS. Further experimental studies are warranted to verify the results of the present study.

\section{Acknowledgements}

The present study was supported by the Shanghai Municipal Commission of Health and Family Planning (grant no. 201540214), and accomplished in Shanghai Key Laboratory of Female Reproductive Endocrine-Related Diseases.

\section{References}

1. Teede H, Deeks A and Moran L: Polycystic ovary syndrome: A complex condition with psychological, reproductive and metabolic manifestations that impacts on health across the lifespan. BMC Med 8: 41, 2010.

2. Kar P and Cummings M: Polycystic ovary syndrome. Pract Diabetes Int 22: 256-260, 2005.

3. Naderpoor N, Shorakae S, Joham A, Boyle J, De Courten B and Teede HJ: Obesity and polycystic ovary syndrome. Minerva Endocrinol 40: 37-51, 2015.

4. Tehrani FR, Simbar M, Tohidi M, Hosseinpanah F and Azizi F: The prevalence of polycystic ovary syndrome in a community sample of Iranian population: Iranian PCOS prevalence study. Reprod Biol Endocrinol 9: 39, 2011.

5. Palioura E and Diamanti-Kandarakis E: Industrial endocrine disruptors and polycystic ovary syndrome. J Endocrinol Invest 36: 1105-1111, 2013.

6. Wang F, Zhang Z, Wang Z, Xiao K, Wang Q, Su J and Wang Z: Expression and clinical significance of the HIF-1 $\alpha /$ ET-2 signaling pathway during the development and treatment of polycystic ovary syndrome. J Mol Histol 46: 173-181, 2015.

7. Ding CF, Chen WQ, Zhu YT, Bo YL, Hu HM and Zheng RH: Circulating microRNAs in patients with polycystic ovary syndrome. Hum Fertil (Camb) 18: 22-29, 2015.

8. Xu B, Zhang YW, Tong XH and Liu YS: Characterization of microRNA profile in human cumulus granulosa cells: Identification of microRNAs that regulate Notch signaling and are associated with PCOS. Mol Cell Endocrinol 404: 26-36, 2015.

9. Kaur S, Archer KJ, Devi MG, Kriplani A, Strauss JF III and Singh R: Differential gene expression in granulosa cells from polycystic ovary syndrome patients with and without insulin resistance: Identification of susceptibility gene sets through network analysis. J Clin Endocrinol Metab 97: E2016-E2021, 2012.

10. Liu HY, Liu JQ, Mai ZX and Zeng YT: A subpathway-based method of drug reposition for polycystic ovary syndrome. Reprod Sci 22: 423-430, 2015.

11. Liu HY, Huang YL, Liu JQ and Huang Q: Transcription factor microRNA synergistic regulatory network revealing the mechanism of polycystic ovary syndrome. Mol Med Rep 13: 3920-3928, 2016. 
12. Bohler A, Wu G, Kutmon M,Pradhana LA, Coort SL, Hanspers K, Haw R, Pico AR and Evelo CT: Reactome from a WikiPathways perspective. PLoS Comput Biol 12: e1004941, 2016.

13. Wu G, Dawson E, Duong A, Haw R and Stein L: ReactomeFIViz: A Cytoscape app for pathway and network-based data analysis. F1000Res 3: 146, 2014

14. Edgar R, Domrachev M and Lash AE: Gene Expression Omnibus: NCBI gene expression and hybridization array data repository. Nucleic Acids Res 30: 207-210, 2002.

15. Mitra PS, Ghosh S, Zang S, Sonneborn D, Hertz-Picciotto I, Trnovec T, Palkovicova L, Sovcikova E, Ghimbovschi S, Hoffman EP, et al: Analysis of the toxicogenomic effects of exposure to persistent organic pollutants (POPs) in Slovakian girls: Correlations between gene expression and disease risk. Environ Int 39: 188-199, 2012.

16. Gautier L, Cope L, Bolstad BM and Irizarry RA: affy - analysis of Affymetrix GeneChip data at the probe level. Bioinformatics 20: 307-315, 2004

17. Gentleman RC, Carey VJ, Bates DM, Bolstad B, Dettling M, Dudoit S, Ellis B, Gautier L, Ge Y, Gentry J, et al: Bioconductor: Open software development for computational biology and bioinformatics. Genome Biol 5: R80, 2004.

18. Liu J, Yang XY and Shi WJ: Identifying differentially expressed genes and pathways in two types of non-small cell lung cancer: Adenocarcinoma and squamous cell carcinoma. Genet Mol Res 13: 95-102, 2014.

19. Szekely GJ and Rizzo ML: Hierarchical clustering via joint between-within distances: Extending Ward's minimum variance method. J Classif 22: 151-183, 2005.

20. Bindea G, Mlecnik B, Hackl H, Charoentong P, Tosolini M, Kirilovsky A, Fridman WH, Pagès F, Trajanoski Z and Galon J: ClueGO: A Cytoscape plug-in to decipher functionally grouped gene ontology and pathway annotation networks. Bioinformatics 25: 1091-1093, 2009.

21. Bindea G, Galon J and Mlecnik B: CluePedia Cytoscape plugin: Pathway insights using integrated experimental and in silico data. Bioinformatics 29: 661-663, 2013.

22. Thissen D and Kuang D: Quick and easy implementation of the Benjamini-Hochberg procedure for controlling the false positive rate in multiple comparisons. J Educ Behav Stat 27: 77-83, 2002.

23. Luo W and Brouwer C: Pathview: An R/Bioconductor package for pathway-based data integration and visualization. Bioinformatics 29: 1830-1831, 2013.

24. Barabási AL, Gulbahce N and Loscalzo J: Network medicine: A network-based approach to human disease. Nat Rev Genet 12: 56-68, 2011.

25. Guzzi PH and Mina M: AlignMCL: Comparative analysis of protein interaction networks through Markov clustering. IEEE International Conference on Bioinformatics and Biomedicine Workshops 174-181, 2012.

26. Joshi-Tope G, Gillespie M, Vastrik I, D'Eustachio P, Schmidt E, de Bono B, Jassal B, Gopinath GR, Wu GR, Matthews L, et al: Reactome: A knowledgebase of biological pathways. Nucleic Acids Res 33: D428-D432, 2005.

27. Schaefer CF, Anthony K, Krupa S, Buchoff J, Day M, Hannay T and Buetow KH: PID: The pathway interaction database. Nucleic Acids Res 37: D674-D679, 2009.

28. Mi $\mathrm{H}$ and Thomas P: PANTHER pathway: An ontology-based pathway database coupled with data analysis tools. Methods Mol Biol 563: 123-140, 2009.

29. Glintborg D and Andersen M: An update on the pathogenesis, inflammation, and metabolism in hirsutism and polycystic ovary syndrome. Gynecol Endocrinol 26: 281-296, 2010.

30. Ojeda-Ojeda M, Murri M, Insenser M and Escobar-Morreale HF: Mediators of low-grade chronic inflammation in polycystic ovary syndrome (PCOS). Curr Pharm Des 19: 5775-5791, 2013.

31. Kovács M, Németh T, Jakus Z, Sitaru C, Simon E, Futosi K, Botz B, Helyes Z, Lowell CA and Mócsai A: The Src family kinases Hck, Fgr, and Lyn are critical for the generation of the in vivo inflammatory environment without a direct role in leukocyte recruitment. J Exp Med 211: 1993-2011, 2014.

32. Scapini P, Pereira S, Zhang H and Lowell CA: Multiple roles of Lyn kinase in myeloid cell signaling and function. Immunol Rev 228: 23-40, 2009.
33. Toubiana J, Rossi AL, Belaidouni N, Grimaldi D, Pene F, Chafey P, Comba B, Camoin L, Bismuth G, Claessens YE, et al: Src-family-tyrosine kinase Lyn is critical for TLR2-mediated $\mathrm{NF}-\kappa \mathrm{B}$ activation through the PI 3-kinase signaling pathway. Innate Immun 21: 685-697, 2015.

34. Wieser V, Moschen AR and Tilg H: Inflammation, cytokines and insulin resistance: A clinical perspective. Arch Immunol Ther Exp (Warsz) 61: 119-125, 2013.

35. DeUgarte CM, Bartolucci AA and Azziz R: Prevalence of insulin resistance in the polycystic ovary syndrome using the homeostasis model assessment. Fertil Steril 83: 1454-1460, 2005.

36. Stankiewicz TR and Linseman DA: Rho family GTPases: Key players in neuronal development, neuronal survival, and neurodegeneration. Front Cell Neurosci 8: 314, 2014.

37. Liu X, Yan F, Yao H, Chang M, Qin J, Li Y, Wang Y and Pei X: Involvement of RhoA/ROCK in insulin secretion of pancreatic $\beta$-cells in 3D culture. Cell Tissue Res 358: 359-369, 2014.

38. Kanda T, Wakino S, Homma K, Yoshioka K, Tatematsu S, Hasegawa K, Takamatsu I, Sugano N, Hayashi K and Saruta T: Rho-kinase as a molecular target for insulin resistance and hypertension. FASEB J 20: 169-171, 2006.

39. Sewer MB and Li D: Regulation of adrenocortical steroid hormone production by RhoA-diaphanous 1 signaling and the cytoskeleton. Mol Cell Endocrinol 371: 79-86, 2013.

40. Vogt DL, Gray CD, Young WS III, Orellana SA and Malouf AT: ARHGAP4 is a novel RhoGAP that mediates inhibition of cell motility and axon outgrowth. Mol Cell Neurosci 36: 332-342, 2007.

41. Kim S, Dangelmaier C, Bhavanasi D, Meng S, Wang H, Goldfinger LE and Kunapuli SP: RhoG protein regulates glycoprotein VI-Fc receptor $\gamma$-chain complex-mediated platelet activation and thrombus formation. J Biol Chem 288: 34230-34238, 2013.

42. Rajendran S, Willoughby SR, Chan WPA, Liberts EA, Heresztyn T, Saha M, Marber MS, Norman RJ and Horowitz JD: Polycystic ovary syndrome is associated with severe platelet and endothelial dysfunction in both obese and lean subjects. Atherosclerosis 204: 509-514, 2009.

43. Stokes KY and Granger DN: Platelets: A critical link between inflammation and microvascular dysfunction. J Physiol 590: 1023-1034, 2012.

44. Salvetti NR, Gimeno EJ, Lorente JA and Ortega HH: Expression of cytoskeletal proteins in the follicular wall of induced ovarian cysts. Cells Tissues Organs 178: 117-125, 2004.

45. Cortón M, Botella-Carretero JI, Benguría A, Villuendas G, Zaballos A, San Millán JL, Escobar-Morreale HF and Peral B: Differential gene expression profile in omental adipose tissue in women with polycystic ovary syndrome. J Clin Endocrinol Metab 92: 328-337, 2007.

46. Ober C, Weil S, Steck T, Billstrand C, Levrant S and Barnes R: Increased risk for polycystic ovary syndrome associated with human leukocyte antigen DQA1"0501. Am J Obstet Gynecol 167: 1803-1806, 1992

47. Kaibe M, Takakuwa K, Murakawa H, Ishii K, Tamura M and Tanaka K: Studies on the human leukocyte antigens in patients with polycystic ovary syndrome in a Japanese population - possible susceptibility of HLA-A11 and -DRB1*0403 to patient population with polycystic ovary syndrome. Am J Reprod Immunol 55: 301-306, 2006.

48. Ozteki O, Fenkci SM, Fenkci V, Enli Y and Cabus U: Serum HLA-G levels in women with polycystic ovary syndrome. Gynecol Endocrinol 31: 243-246, 2015.

49. Strodthoff D, Ma Z, Wirström T, Strawbridge RJ, Ketelhuth DF, Engel D, Clarke R, Falkmer S, Hamsten A, Hansson GK, et al: Toll-like receptor 3 influences glucose homeostasis and $\beta$-cell Insulin Secretion. Diabetes 64: 3425-3438, 2015.

50. Hemmati F, Ghasemi R, Mohamed Ibrahim N, Dargahi L, Mohamed Z, Raymond AA and Ahmadiani A: Crosstalk between insulin and Toll-like receptor signaling pathways in the central nervous system. Mol Neurobiol 50: 797-810, 2014. 